\title{
Interference Alignment: Improved design via precoding vectors
}

\author{
Yasser Fadlallah, Abdeldjalil Aïssa-El-Bey, Karine Amis and Ramesh Pyndiah \\ Institut Télécom; Télécom Bretagne; UMR CNRS 3192 Lab-STICC \\ Technopôle Brest Iroise CS 8381829238 Brest, France \\ Université européenne de Bretagne \\ Email: Firstname.Lastname@telecom-bretagne.eu
}

\begin{abstract}
The degree of freedom of the Single Input Single Output (SISO) fading interference channel is asymptotically upperbounded by $\mathrm{K} / 2$. This upperbound can be achieved using the Interference Alignment approach (IA), proposed by Cadambe et al. In this work, a new optimized design of the IA scheme is presented. It involves introducing, for each user, a combination matrix so as to maximize the sum rate of the network. The optimal design is obtained via an iterative algorithm proposed in the $K$-user IA network, and a convergence to a local optimum is achieved. Numerical results enable us to evaluate the performance of the new algorithm and to compare it with other designs.
\end{abstract}

\section{INTRODUCTION}

A few years ago, the capacity region of the fading interference channels was an open problem. Several researches have pursued some special cases among which [1]-[3]. One of them is the two-user $X$ channel, which has been studied in [3], and a new upperbound has been obtained. The capability of achieving this upperbound involves introducing a new approach of interference management, known as Interference Alignment (IA)

The basic idea of the IA is to design transmitted signals such that interfering signals at each receiver overlap while the desired signal remains distinct from interferences. Cadambe and Jafar (CJ) have exploited this approach in order to show that the maximum achieved Degrees of Freedom (DoF) for $K$ user time-varying interference channels, in the $n$-dimensional Euclidean space, is $\frac{K}{2}$ [4]. Inspired by the IA idea for time-varying fading interference channels, many efforts have been made to extend this idea to the quasi-static interference channels [5], [6]. Considering a transmission with quasi-static real channel coefficients, Motahari et al. have demonstrated the capability to achieve the full DoF in the one-dimensional spaces (rational spaces) using the IA approach [7].

A common assumption in the aforementioned schemes is that the users are synchronous and the received signal at each receiver node is a synchronized linear combination of the transmitted signals. In practice, such an assumption is not realistic. Therefore, the asynchronous transmission in the $K$ user fading interference channels with quasi-static coefficients is considered in [8]. It has been shown that the total DoF of this channel is equal to the one of the corresponding synchronous channel. The full DoF is achieved using a novel IA scheme with no need of any channel state information at the transmitters.
In the IA schemes described above, the full DoF is achieved for large dimensional precoding matrices, and for signal-tonoise ratio (SNR) close to infinity. The maximum achievable DoF is defined as

$$
\lim _{S N R \rightarrow \infty} \frac{C(S N R)}{\log _{2}(S N R)}=\frac{K}{2},
$$

where $K$ is the total user number in the fading interference channel, and $C(S N R)$ represents the channel capacity.

In order to optimize the IA beamforming design and to improve the data rate performance, some methods have been proposed such as in [9]-[11]. One of these methods suggests to introduce a combination matrix at each transmitter, and optimize it so as to maximize the sum rate for high signalto-noise ratio (SNR), under the assumption of Zero-Forcing (ZF) decoding scheme. The optimal solution of the introduced matrix is the one that orthonormalizes the precoding matrix for each user. In this paper we aim to maximize the sum rate of the IA network employing a Minimum Mean Square Error (MMSE) decoding scheme and assuming synchronous users. Compared to the ZF decoder, the main advantage of an MMSE decoder is a better error performance while a matched filter receiver can be implemented at lower computational cost. An MMSE shows an equivalent error performance to a $\mathrm{ZF}$ for high SNR level.

This paper is organized as follows. In Section II, we describe the system model of the $K$-user fading interference channel. Section III reviews some related work to highlight our contribution. The proposed optimization algorithm is given in Section IV. Numerical results are shown in the $K$-user SISO fading interference channel in Section V. Finally, Section VI concludes the paper.

Notations: boldface upper case letters and boldface lower case letters denote matrices and vectors, respectively. For the transpose, transpose conjugate and conjugate matrix we use $(.)^{t},(.)^{H}$ and $(.)^{*}$, respectively. $|$.$| and \operatorname{tr}($.$) denote the$ determinant the trace of a matrix, respectively. The vec(.) operator indicates the vectorization of a matrix, and $\mathrm{J}($. denotes the Jacobian of a matrix.

\section{SYSTEM MODEL}

Consider the $K$-user SISO time-varying block fading interference channel as illustrated in Fig. 1, with $K$ transmit receive 
pairs. A wireless channel links each receiver to one transmitter, and each transmitter intends to have its signal decoded by its destination. The proposed IA scheme in [12] shows more efficiency than the CJ scheme for $K>3$, and higher DoF is achieved for reduced channel extension. A synchronized scheme is adopted with perfect channel knowledge at each source and destination ${ }^{1}$. The precoding matrices in this model are chosen in order to satisfy the IA scheme and to maximize the DoF for limited channel extension, simultaneously [12]. The DoF per user is obtained using the following combinations

$$
d^{1}=\left(\begin{array}{c}
m^{*}+M+1 \\
M
\end{array}\right) \quad \text { and } \quad d^{3}=\left(\begin{array}{c}
m^{*}+M \\
M
\end{array}\right)
$$

where $m^{*}$ is a given nonnegative integer, $M$ is a parameter depending on the user number $M=(K-1)(K-2)-1$, and $d^{i}$ is the DoF of the $i^{t h}$ user. Provided $d^{i}=d^{3}, d^{1}>$ $d^{3}, i \in \mathcal{K} \backslash\{1,3\}$, IA can be then satisfied. $\mathcal{K}$ represents the set of the user indeces $\{1,2, \ldots, K\}$. The precoding matrix length, obtained using the channel extensions, is given as $N=$ $d^{1}+d^{2}$. The channel is supposed to be frequency selective, and the Orthogonal Frequency Division Multiplexing (OFDM) transmission technique is applied (in this case $N$ denotes the subcarrier number). At the $k^{t h}$ destination, the channel output is given by

$$
\boldsymbol{y}_{k}=\sum_{j=1}^{K} \boldsymbol{H}_{k j} \boldsymbol{V}_{j} \boldsymbol{x}_{j}+\boldsymbol{z}_{k}, \forall k \in \mathcal{K},
$$

where $\boldsymbol{H}_{k j}$ is the $N \times N$ diagonal channel fading matrix between the $j^{t h}$ transmitter and the $k^{t h}$ receiver. $\boldsymbol{V}_{j}$ is the $N \times d^{j}$ precoding matrix of the $j^{t h}$ transmitter. The $j^{t h}$ transmitted information $\boldsymbol{x}_{j}$ is defined as a $d^{j} \times 1$ vector. $\boldsymbol{z}_{k}$ is the $N \times 1$ circular symmetric complex Gaussian noise vector at the receiver $k$, with independent and identically distributed (i.i.d.) components; i.e. $\boldsymbol{z}_{k} \sim \mathcal{N}_{c}\left(0, \boldsymbol{I}_{N}\right)$.

According to [12], the beamforming design criterion maximizing the DoF of the IA scheme is defined as

$$
\begin{array}{r}
\boldsymbol{V}_{1}=\left\{\prod_{k, l \in \mathcal{K} \backslash 1, k \neq l,(k, l) \neq(2,3)}\left(\left(\boldsymbol{T}_{23}\right)^{-1} \boldsymbol{T}_{k l}\right)^{n_{k l}} \boldsymbol{w}\right. \\
\left.\mid \sum_{k, l \in \mathcal{K} \backslash 1, k \neq l,(k, l) \neq(2,3)} n_{k l} \leq m^{*}+1\right\}
\end{array}
$$

and

$$
\begin{array}{r}
\boldsymbol{V}_{3}=\left\{\left(\boldsymbol{T}_{23}\right)^{-1} \prod_{k, l \in \mathcal{K} \backslash 1, k \neq l,(k, l) \neq(2,3)}\left(\left(\boldsymbol{T}_{23}\right)^{-1} \boldsymbol{T}_{k l}\right)^{n_{k l}} \boldsymbol{w}\right. \\
\left.\sum_{k, l \in \mathcal{K} \backslash 1, k \neq l,(k, l) \neq(2,3)} n_{k l} \leq m^{*}\right\},
\end{array}
$$

where $\boldsymbol{w}$ is the $N \times 1$ vector determining the beamforming vectors of each user. For an optimized data rate performance, $\boldsymbol{w}$ should be judiciously chosen in order to maximize the data rate as described in [9], [10]. $\boldsymbol{T}_{k l}$ is an $N \times N$ diagonal matrix defined as

$$
\boldsymbol{T}_{k l}=\left(\boldsymbol{H}_{k 1}\right)^{-1} \boldsymbol{H}_{k l}\left(\boldsymbol{H}_{1 l}\right)^{-1} \boldsymbol{H}_{13} .
$$

\footnotetext{
${ }^{1}$ It is important also to recall that this scheme can be easily extended to
} the one proposed in the asynchronous transmission (cf. Section IV in [8]).

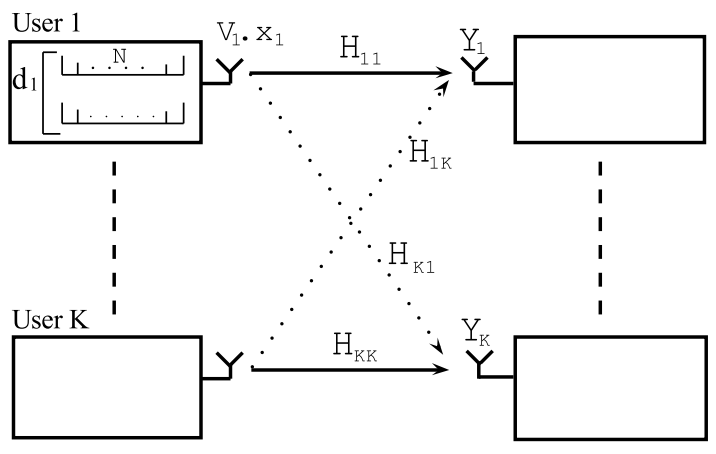

Fig. 1. The $K$-user SISO fading interference channel

In the remaining of the paper, the precoder is modified by introducing a combination matrix at each transmitter. Thus in (2), the matrix $\boldsymbol{V}_{k}$ is replaced by a matrix $\boldsymbol{P}_{k}$ defined as

$$
\boldsymbol{P}_{k}=\boldsymbol{V}_{k} \cdot \boldsymbol{C}_{k},
$$

where $\boldsymbol{C}_{k}$ is a $d^{k} \times d^{k}$ matrix that will be designed so as to maximize the sum rate while keeping the IA scheme satisfied, and thus, ensuring a full asymptotically achievable DoF for the network.

\section{REVIEW WORKS ABOUT THE IA BEAMFORMING OPTIMIZED DESIGNS}

In this section, we review the two algorithms that seek to find the combination matrices $\boldsymbol{C}_{k}$, for all $k$, that maximize the data rate performance. We also recall the expression of the maximum individual rate for an MMSE receiver.

\section{A. Optimization of the precoding vectors}

The optimization of the precoding vectors within the subspaces, proposed by $\mathrm{CJ}$ at each transmitter, was firstly proposed in [11]. This perspective aims to maximize, in the 3 user SISO fading interference channel, the high SNR offset of the sum rate under power limitation constraints (cf. eq. (5) and (8)-(20) in [11]) while preserving the full asymptotically achievable DoF. The optimized precoders is given by

$$
\begin{aligned}
& \tilde{\boldsymbol{V}}_{1}=\frac{\boldsymbol{V}_{1}}{\left\|\boldsymbol{V}_{1}\right\|} \\
& \tilde{\boldsymbol{V}}_{2}=\boldsymbol{V}_{2} \boldsymbol{F}, \\
& \tilde{\boldsymbol{V}}_{3}=\boldsymbol{V}_{3} \boldsymbol{E} .
\end{aligned}
$$

Considering the optimization of the high SNR offset of the sum rate for a $\mathrm{ZF}$ receiver, the maximum solutions of $\boldsymbol{E}$ and $\boldsymbol{F}$ are $d^{k} \times d^{k}$ matrices that orthonormalizing the $d^{k}$ columns of $\boldsymbol{V}_{2}$ and $\boldsymbol{V}_{3}$, respectively, while allocating an average power of $\frac{N}{d^{k}}$ for each stream of the $k^{t h}$ transmitter.

Unfortunately, the ZF increases the level noise at the reception leading to a degradation error performance. Therefore MMSE criterion has been employed by Sung et al. and the optimization problem has been proposed throughout the SNR region [13]. However, a suboptimal strategy was shown where the $C_{k}$, at each transmitter, has been chosen as a unitary matrix in order to reduce the computational complexity. 


\section{B. Maximum sum rate expression}

The proposed algorithm in this paper is based on the sum rate expression that depends on the MMSE decoding matrix. Therefore, the decoding scheme of each user must be designed carefully so as to maximize the information rate for all users. The expression of the maximum individual information rate for an MMSE receiver is obtained as

$$
R_{k}=\log _{2} \frac{\left|\boldsymbol{I}+p \sum_{j=1}^{K} \boldsymbol{H}_{k j} \boldsymbol{P}_{j} \boldsymbol{P}_{j}^{H} \boldsymbol{H}_{k j}^{H}\right|}{\left|\boldsymbol{I}+p \sum_{j \neq k}^{K} \boldsymbol{H}_{k j} \boldsymbol{P}_{j} \boldsymbol{P}_{j}^{H} \boldsymbol{H}_{k j}^{H}\right|} .
$$

Based on this information rate expression, a new improved design for the IA scheme is proposed in the upcoming section.

\section{ITERATIVE MAXIMIZATION OF THE SUM RATE FUNCTION}

\section{A. Iterative algorithm}

Maximizing the sum rate function requires finding the optimal precoding vectors of each precoder subspaces, therefore a new matrix $\boldsymbol{C}_{k}$ is combined with each precoding matrix $\boldsymbol{V}_{k}$. A closed-form solution of this combined matrix is nontrivial. Therefore, we seek to find the solution iteratively using the sum rate maximization criterion. Using the MMSE maximum information rate in (8), the sum rate maximization problem, constrained by the individual power limitation, is formulated as follows

$$
\underset{\boldsymbol{C}_{k}, k \in \mathcal{K}}{\operatorname{argmax}} \sum_{k=1}^{K} \frac{1}{N} R_{k}=
$$

$\underset{\boldsymbol{C}_{k}, k \in \mathcal{K}}{\operatorname{argmax}} \frac{1}{N} \sum_{k=1}^{K} \log _{2} \frac{\left|\boldsymbol{I}+p \sum_{j=1}^{K} \overline{\boldsymbol{H}}_{k j} \boldsymbol{C}_{j}\left(\overline{\boldsymbol{H}}_{k j} \boldsymbol{C}_{j}\right)^{H}\right|}{\left|\boldsymbol{I}+p \sum_{j \neq k}^{K} \overline{\boldsymbol{H}}_{k j} \boldsymbol{C}_{j}\left(\overline{\boldsymbol{H}}_{k j} \boldsymbol{C}_{j}\right)^{H}\right|}$

$$
\text { subject to } \quad \operatorname{tr}\left(\boldsymbol{V}_{k} \boldsymbol{C}_{k} \boldsymbol{C}_{k}^{H} \boldsymbol{V}_{k}^{H}\right)=N, k \in \mathcal{K} .
$$

with $\overline{\boldsymbol{H}}_{k j}=\boldsymbol{H}_{k j} \boldsymbol{V}_{j}$. Due to the variation of the matrices $\boldsymbol{C}_{k}$ at each iteration, for all $k$, the considered problem is non concave, which results in a local optimal solution. This kind of constrained problem can be solved by transforming it to an unconstrained problem and then applying a first order optimization method such as the gradient descent method. Other methods can also be used such as Newton method. However, a higher computation cost is required. Let us start by defining the matrix $\boldsymbol{C}_{k}$ as

$$
\boldsymbol{C}_{k}=\sqrt{\alpha_{k}} \overline{\boldsymbol{C}}_{k}, \quad \text { with } \alpha_{k}=\frac{1}{\operatorname{tr}\left(\boldsymbol{V}_{k} \overline{\boldsymbol{C}}_{k} \overline{\boldsymbol{C}}_{k}^{H} \boldsymbol{V}_{k}^{H}\right)} .
$$

Substituting (10) into (9) and considering $\overline{\boldsymbol{C}}_{k}$ as the new variable, the constraint in (9) is satisfied for any $\overline{\boldsymbol{C}}_{k}$ and the resulting optimization problem gets unconstrained. The optimization problem is now formulated as

$\underset{\overline{\boldsymbol{C}}_{k}, k \in \mathcal{K}}{\operatorname{argmax}} R ; R \equiv \sum_{k=1}^{K} \log _{2} \frac{\left|\boldsymbol{I}+p \sum_{j=1}^{K} \alpha_{j} \overline{\boldsymbol{H}}_{k j} \overline{\boldsymbol{C}}_{j}\left(\overline{\boldsymbol{H}}_{k j} \overline{\boldsymbol{C}}_{j}\right)^{H}\right|}{\left|\boldsymbol{I}+p \sum_{j \neq k}^{K} \alpha_{j} \overline{\boldsymbol{H}}_{k j} \overline{\boldsymbol{C}}_{j}\left(\overline{\boldsymbol{H}}_{k j} \overline{\boldsymbol{C}}_{j}\right)^{H}\right|}$
The expression of the sum rate can be rewritten in the following compact form

$$
R \equiv \sum_{k=1}^{K} \log _{2}\left|\boldsymbol{X}_{k}\right|-\log _{2}\left|\boldsymbol{Y}_{k}\right|
$$

with

$$
\begin{aligned}
\boldsymbol{X}_{k} & =\boldsymbol{I}+p \sum_{j=1}^{K} \alpha_{j} \overline{\boldsymbol{H}}_{k j} \overline{\boldsymbol{C}}_{j}\left(\overline{\boldsymbol{H}}_{k j} \overline{\boldsymbol{C}}_{j}\right)^{H} \\
\boldsymbol{Y}_{k} & =\boldsymbol{I}+p \sum_{j \neq k}^{K} \alpha_{j} \overline{\boldsymbol{H}}_{k j} \overline{\boldsymbol{C}}_{j}\left(\overline{\boldsymbol{H}}_{k j} \overline{\boldsymbol{C}}_{j}\right)^{H}
\end{aligned}
$$

For such a problem, the gradient descent algorithm which has a simple implementation, could be applied. However, it takes too many iterations to converge. The optimal $\bar{C}_{k}$ is obtained using the Jacobian of $R\left(\overline{\boldsymbol{C}}_{k}\right)$; function of $\overline{\boldsymbol{C}}_{k}$. This Jacobian can be computed from the differential of the function $R \bar{C}_{k}$ as described in [14]. As $\overline{\boldsymbol{C}}_{k}$ is a complex matrix, then $\mathrm{d} R=$ $2 \partial R / \overline{\boldsymbol{C}}_{k}^{*}$. The differential of $\log _{2}\left|\boldsymbol{X}_{k}\right|$ is computed as

$$
\mathrm{d} \log _{2}\left|\boldsymbol{X}_{k}\right|=\operatorname{tr}\left(\boldsymbol{X}_{k}^{-1} d \boldsymbol{X}_{k}\right)
$$

$\mathrm{d} \boldsymbol{X}_{k}=p \alpha_{k} \overline{\boldsymbol{H}}_{k j} \overline{\boldsymbol{C}}_{j} \mathrm{~d} \overline{\boldsymbol{C}}_{j}^{H} \overline{\boldsymbol{H}}_{k j}^{H}+p \mathrm{~d} \alpha_{k} \overline{\boldsymbol{H}}_{k j} \overline{\boldsymbol{C}}_{j} \overline{\boldsymbol{C}}_{j}^{H} \overline{\boldsymbol{H}}_{k j}^{H}$,

Using the property $\operatorname{tr}\left(\boldsymbol{A} d \boldsymbol{B}^{H}\right)=\operatorname{tr}\left(\boldsymbol{A}^{t} d \boldsymbol{B}^{*}\right)$ and referring to [14] that describes the first-order differentials and the Jacobian matrix properties, we obtain

$$
\begin{array}{r}
\mathrm{d} \log _{2}\left|\boldsymbol{X}_{k}\right|=\frac{2 p}{\ln 2}\left[\alpha_{k} \operatorname{vec}\left(\overline{\boldsymbol{H}}_{k k}^{H} \boldsymbol{X}_{k}^{-1} \overline{\boldsymbol{H}}_{k k} \overline{\boldsymbol{C}}_{k}\right)^{t}\right. \\
\left.-\alpha_{k}^{2} \operatorname{tr}\left(\overline{\boldsymbol{C}}_{k}^{H} \overline{\boldsymbol{H}}_{k k}^{H} \boldsymbol{X}_{k}^{-1} \overline{\boldsymbol{H}}_{k k} \overline{\boldsymbol{C}}_{k}\right) \operatorname{vec}\left(\boldsymbol{V}_{k}^{H} \boldsymbol{V}_{k} \overline{\boldsymbol{C}}_{k}\right)^{t}\right] \operatorname{vec}\left(\mathrm{d} \overline{\boldsymbol{C}}_{k}^{*}\right) .
\end{array}
$$

Thus, the derivative of $R$ with respect to $\overline{\boldsymbol{C}}_{k}$ is obtained as follows

$$
\begin{gathered}
\mathrm{J}\left(R\left(\overline{\boldsymbol{C}}_{k}\right)\right)=\frac{2 p}{\ln 2} \alpha_{k} \sum_{i=1}^{K} \overline{\boldsymbol{H}}_{i k}^{H} \boldsymbol{X}_{i}^{-1} \overline{\boldsymbol{H}}_{i k} \overline{\boldsymbol{C}}_{k} \\
-\frac{2 p}{\ln 2} \alpha_{k}^{2} \sum_{i=1}^{K} \operatorname{tr}\left[\overline{\boldsymbol{C}}_{k}^{H} \overline{\boldsymbol{H}}_{i k}^{H} \boldsymbol{X}_{i}^{-1} \overline{\boldsymbol{H}}_{i k} \overline{\boldsymbol{C}}_{k}\right] \boldsymbol{V}_{k}^{H} \boldsymbol{V}_{k} \overline{\boldsymbol{C}}_{k} \\
-\frac{2 p}{\ln 2} \alpha_{k} \sum_{i \neq k}^{K} \overline{\boldsymbol{H}}_{i k}^{H} \boldsymbol{Y}_{i}^{-1} \overline{\boldsymbol{H}}_{i k} \overline{\boldsymbol{C}}_{k} \\
-\frac{2 p}{\ln 2} \alpha_{k}^{2} \sum_{i \neq k}^{K} \operatorname{tr}\left[\overline{\boldsymbol{C}}_{k}^{H} \overline{\boldsymbol{H}}_{i k}^{H} \boldsymbol{Y}_{i}^{-1} \overline{\boldsymbol{H}}_{i k} \overline{\boldsymbol{C}}_{k}\right] \boldsymbol{V}_{k}^{H} \boldsymbol{V}_{k} \overline{\boldsymbol{C}}_{k} .
\end{gathered}
$$

Using this derivative, the matrix $\overline{\boldsymbol{C}}_{k}$ at the $k^{t h}$ transmitter is iteratively computed as described in the following algorithm:

1) Fix the $d^{k} \times d^{k}$ matrix $\overline{\boldsymbol{C}}_{k}^{(0)}$ to the identity matrix for all $k$

2) for $\mathrm{k}=1: \mathrm{K}$

- Calculate $\mathbf{J}\left(R\left(\overline{\boldsymbol{C}}_{k}\right)\right)$; the Jacobian of $R$.

- Update $\overline{\boldsymbol{C}}_{k}^{(l+1)}=\overline{\boldsymbol{C}}_{k}^{(l)}+\mu \nabla_{\overline{\boldsymbol{C}}_{k}^{(l)}} R$.

3) Iterate step 2 until convergence

In this algorithm, the step size $\mu$ is a determining factor to ensure a faster convergence, thus, it must be judiciously 
selected. In [15], two line search methods are proposed: exact line search and inexact line search methods. Most line searches are inexact in practice, and many methods have been proposed. Herein, the backtracking line search method is used, which is very simple to implement and quite effective. The stop criterion of this iterative algorithm is supposed to be achieved either when

$$
\sum_{k}\left\|\mathbf{J}\left(R\left(\overline{\boldsymbol{C}}_{k}\right)\right)\right\|<\epsilon
$$

or when a maximum number of iterations is achieved, where $\epsilon$ is defined as a tolerence factor.

The main drawback in the above algorithm is that the convergence to a global maximum is not ensured. However, as long as the gradient descent method is applied, the matrix $\overline{\boldsymbol{C}}_{k}$ will move in the direction of the Jacobian, which could imply a convergence to a local maximum. The convergence will be illustrated in the next section.

\section{B. Extension to the asynchronous system}

In the IA optimized scheme above, all users are supposed synchronous and the signal at each receiver is a linear of the synchronized transmitted signals. Unfortunately, this seems to be an unrealistic assumption, where propagation delays appear even though synchronization providers exist. Therefore, an extension to the asynchronous interference alignment scheme, proposed by [8] in the quasi-static fading interference channel, is shown.

Referring to the equation (37) in [8], the optimized design proposed above can be applied directly to the IA scheme in the asynchronous network. In this scheme, there is no need of any channel knowledge at the transmitters. However, the relative delays between all transmitters and receivers are required to be estimated. Therefore, to adapt our optimized scheme to the asynchronous case, we have to replace the channel matrix $\boldsymbol{H}_{k j}$ by the circulant convolution matrix $\boldsymbol{\Gamma}_{k j}$ (equation (13) in [8]) that depends on the values of the relative asynchronous delay.

\section{NUMERICAL RESULTS}

In this section, we present the numerical results of the improved design, derived in IV, in the 3-user SISO fading interference channel. The exhibited results are based on 1000 channel realizations. Channel coefficients have an i.i.d. circular symmetric complex Gaussian distribution with zero mean and unit variance. In the next figures and interpretations, the following abbreviations are used

- CJ- The IA scheme proposed by Cadambe and Jafar [4]

- SHV- The IA Improved design proposed by Shen, HostMadsen and Vidal reviewed in Section III

- Cnew- The IA design with the improvement proposed in this paper

- $D_{n} \log (s n r)$ - The maximum DoF for high SNR with $n=$ $m^{*}+1$

Depending on $n, D_{n}$ is computed as

$$
D_{n}=\frac{d_{1}+d_{2}}{\sum_{k=1}^{K} d_{k}},
$$

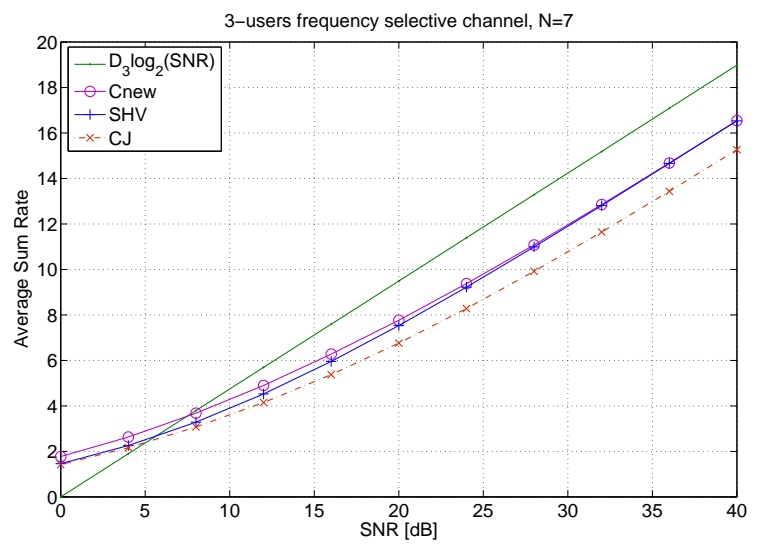

Fig. 2. Average sum rate performance comparison between the different designs for $K=3$ and $N=7$ assuming an MMSE receiver

where $d_{k}$ is given in (2) (cf. Section II). In all the above designs, we fix $\boldsymbol{w}$ as defined by the equation (29) in [10]. The CJ design is the IA scheme with precoding matrices defined in (4). In the following results, the data rate performance of the following designs: CJ, SHV and Cnew, are compared for different values of $N$. For the Cnew design, the maximum is supposed to be achieved either when

$$
\left\|\mathbf{J}\left(R\left(\overline{\boldsymbol{C}}_{k}\right)\right)\right\|<\epsilon
$$

where $\epsilon$ is the tolerance factor for stopping the iterations, or when a maximum number of iterations is achieved. The step size is chosen using the backtracking line search method as in [15] to provide provable convergence.

In Fig. 2, it is clear that the combination with a new matrix while preserving the IA scheme, improves the data rate performance of the network. For $n=3$, the Cnew design outperforms the CJ design by $0.35-0.47$ bits $/ \mathrm{s} / \mathrm{Hz}$ for $\mathrm{SNR}$ values comprised between $0 \mathrm{~dB}$ and $8 \mathrm{~dB}$. This gain increases with the SNR. However, compared to the design with SHV combination, which optimizes the IA scheme for high SNR, we observe that the gain of the proposed design towards the SHV design decreases as the SNR increases. For the SNR values between $0 \mathrm{~dB}$ and $8 \mathrm{~dB}$, a gain of about $0.35 \mathrm{bits} / \mathrm{s} / \mathrm{Hz}$ is shown over the SHV design. However, this gain decreases with SNR to achieve $0.01 \mathrm{bits} / \mathrm{s} / \mathrm{Hz}$ at $40 \mathrm{~dB}$.

Increasing the DoF to $n=5$, a higher gain between these different designs is shown. The Cnew design outperforms the $\mathrm{CJ}$ by a $0.45-0.84$ bits $/ \mathrm{s} / \mathrm{Hz}$ between $0 \mathrm{~dB}$ and $8 \mathrm{~dB}$ as illustrated in Fig. 3. Increasing the SNR value to $20 \mathrm{~dB}$, a gain of about $1.56 \mathrm{bits} / \mathrm{s} / \mathrm{Hz}$ is obtained. Similar improvements are achieved over the SHV design. The average sum rate gain is 0.38 bits $/ \mathrm{s} / \mathrm{Hz}$ at $0 \mathrm{~dB}$ and $0.57 \mathrm{bits} / \mathrm{s} / \mathrm{Hz}$ at $16 \mathrm{~dB}$. The sum rate gain compared with the SHV design increases with SNR until the SNR value of $16 \mathrm{~dB}$ is achieved, and then decreases with SNR to achieve the same performance at high SNR levels. Moreover, the difference for low and mid SNR regime increases with $N$. These simulation results show that 


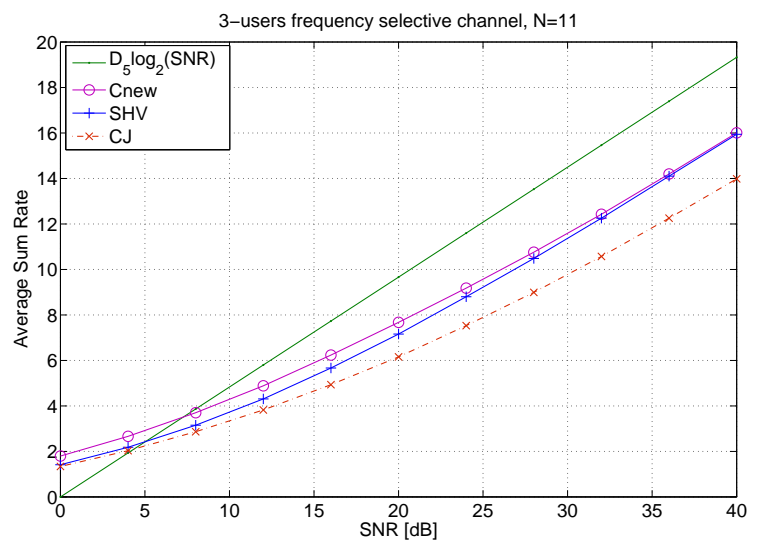

Fig. 3. Average sum rate performance comparison between the different designs for $K=3$ and $N=11$ assuming an MMSE receiver

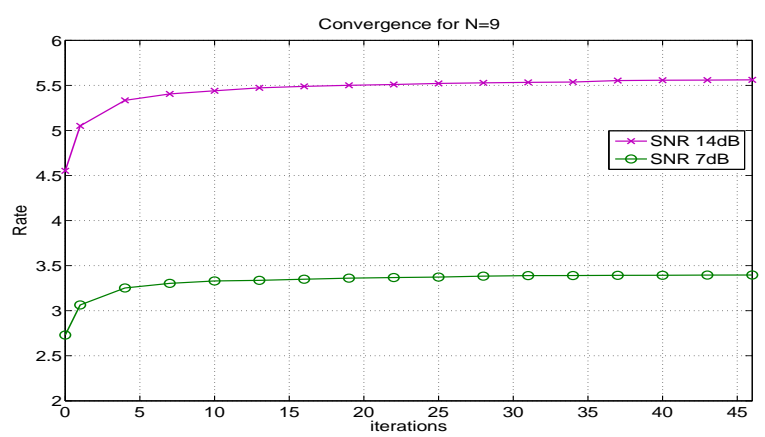

Fig. 4. Average sum rate convergence using the new design for $N=9$ at different SNR values

the new design outperforms the SHV design for low to mid SNR values, and performs the same in the high SNR regime.

Finally, the provable convergence of the proposed iterative algorithm is illustrated in Fig. 4. The sum rate of the new design grows up with the number of iterations while an improvement in performance at each additional iteration is observed. Comparing the convergence at $7 \mathrm{~dB}$ and $14 \mathrm{~dB}$, we observe that more iterations are required for higher SNR values while higher gap between the optimal design and the non optimized design is obtained. The number of iterations could be reduced with a better initialization.

\section{CONCLUSION}

In this paper, a new algorithm is proposed in order to improve the data rate performance of the $K$-user SISO fading synchronous interference channel. This improvement is achieved by optimizing the precoding vectors for each user. It consists in introducing a combination matrix at each transmitter while preserving the IA scheme. The introduced matrices are computed using the gradient descent method. However, the non concavity of the problem makes the solution converge to a local optimal. Numerical results illustrate the performance of the proposed algorithm, which is then compared to other optimized designs. It outperforms existing schemes in the targeted low to medium SNR regime and performs the same in the high SNR regime. This scheme can be easily extended to an asynchronous user scheme and to the MIMO case described in [8].

\section{REFERENCES}

[1] H. Sato, "On degraded gaussian two-user channels," IEEE Transactions on Information Theory, vol. IT-24, pp. 637-640, Sept. 1978.

[2] G. Kramer, "Outer bounds on the capacity of gaussian interference channels," IEEE Transactions on Information Theory, vol. 50, pp. 581586, Mar. 2004.

[3] M. Maddah-Ali, A. S. Motahari, and A. K. Khandani, "Communication over MIMO X channels: interference alignment, decomposition, and performance analysis," IEEE Transactions on Information Theory, vol. 54, pp. 3457-3470, Aug. 2008.

[4] V. R. Cadambe and S. A. Jafar, "Interference alignment and degrees of freedom of the K-user interference channel," IEEE Transactions on Information Theory, vol. 54, no. 8, Aug. 2008.

[5] V. R. Cadambe and S. A. Jafar and C. Wang, "Interference alignment with asymmetric complex signaling: settling the Host-MadsenNosratinia conjecture," IEEE Transactions on Information Theory, vol. 56, no. 9, Sept. 2010.

[6] V. R. Cadambe and S. A. Jafar, "On the degrees of freedom of the K-user Gaussian interference channel," arXiv:0901.1695v1.

[7] A. S. Motahari, S. O. Gharan, and A. K. Khandani, "Real interference alignment with real numbers," arXiv:0908.1208v2, Aug. 2009.

[8] M. Torbatian, H. Najafi, and O. Damen, "Asynchronous interference channel: Degrees of freedom and interference alignment," http://arxiv.org/abs/1101.0275v1, Dec. 2010.

[9] D. Kim and M. Torlak, "Optimization of interference alignment beamforming vectors," IEEE Journal on Selected Areas In Communications, vol. 28, no. 9, pp. 1425-1434, Dec. 2010.

[10] D. Kim and M. Torlak, "Interference alignment via improved subspace conditioning," in Proc. of IEEE Globecom, 2010.

[11] M. Shen, A. Host-Madsen, and J. Vidal, "An improved interference alignment scheme for frequency selective channels," in Proc. of IEEE International Symposium on Information Theory, July 2008, pp. 6-11.

[12] S. W. Choi, S. A. Jafar, and S.-Y. Chung, "On the beamforming design for interference alignment," IEEE Communication Letter, vol. 13, no. 11, pp. 847-849, Nov. 2009.

[13] H. Sung, S. Park, K. Lee, and I. Lee, "Linear precoder designs for k-user interference channels," IEEE Transactions on Wireless Communications, vol. 9, no. 1, pp. 291-300, Jan. 2010.

[14] J. Magnus and H. Neudecker, Matrix Differential Calculus with Applications in Statistics and Econometrics. JHON WILEY SONS, revised version 2007.

[15] S. Boyd and L. Vandenberghe, Convex Optimization. Cambridge University Press, New York, 2004. 